\title{
MODELO LOGÍSTICO EXPLICATIVO DE LAS RELACIONES ENTRE AUTOCONCEPTO Y
} RENDIMIENTO ACADÉMICO

A LOGISTIC EXPLANATORY MODEL OF THE RELATIONSHIP BETWEEN SELF-CONCEPT AND ACADEMIC PERFORMANCE

\author{
ANTONIO HUMBERTO CLOSAS \\ NOELIA BEATRIZ FRANCHINI \\ LUCIANA CYNTHIA KUC \\ MARÍA ALICIA DUSICKA \\ CARLOS MATÍAS HISGEN
}

Facultad de Ciencias Económicas

Universidad Nacional del Nordeste

ARGENTINA

A medida que empecé a quererme, dejé de ansiar tener una vida diferente, y pude ver que todo lo que me rodeaba me estaba invitando a crecer.

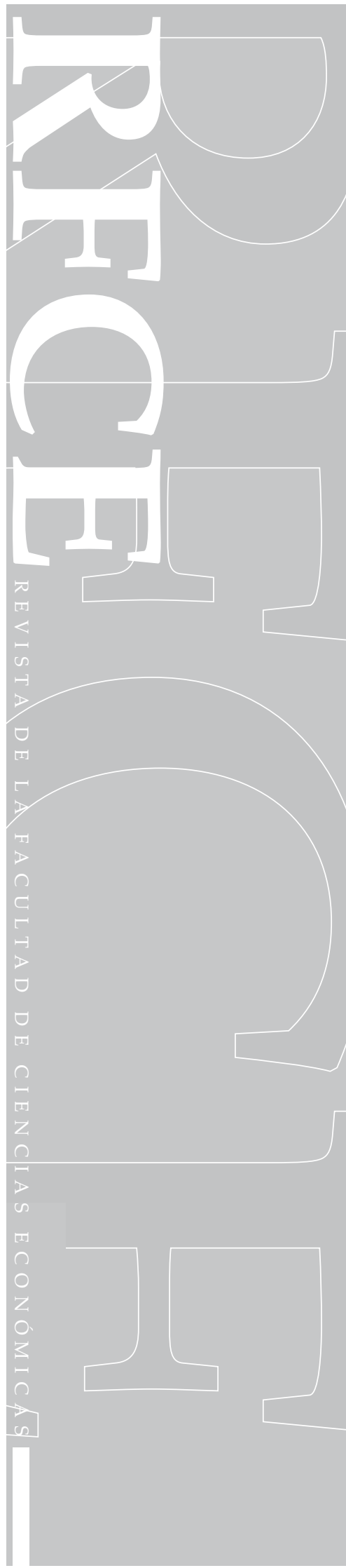




\section{RESUMEN}

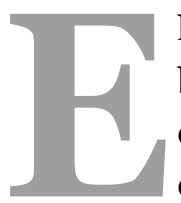

1 rendimiento académico representa un fenómeno multidimensional, que es posible abordarlo tomando en cuenta distintos factores, sus vinculaciones y las implicancias que en él pueden tener. En este contexto, el objetivo principal de nuestro estudio fue desarrollar un modelo de regresión logística que permita explicar de qué manera distintas áreas del constructo autoconcepto se relacionan con los resultados académicos. La muestra, formada por alumnos que pertenecen a una Universidad Nacional (de gestión pública) de Argentina, resultó elegida de manera estratificada, por conglomerados y de forma aleatoria; la misma está compuesta por 164 jóvenes (91 mujeres y 73 hombres), con una media de 19.80 años $(D E=1.72)$. La investigación responde a un diseño explicativo, de estilo descriptivo mediante encuesta (se utilizó el test Autoconcepto Forma 5: Académica, Social, Emocional, Familiar y Física), de línea cuantitativa y de corte transversal. En la etapa empírica, los análisis estadísticos descriptivos, psicométricos e inferenciales, permitieron conocer ciertas características de las dimensiones de la prueba, los índices de consistencia interna de las diferentes áreas y del instrumento en su conjunto, así como determinar el modelo logístico que mejor se ajusta a los datos muestrales. El concepto de la curva ROC ha sido empleado con el fin de mostrar la capacidad global que el modelo posee para explicar los resultados del rendimiento académico. En definitiva, se puede sostener que el cuestionario aplicado es una prueba confiable, que posee validez predictiva para describir la variabilidad de los resultados académicos a partir de los distintos tipos de autoconcepto analizados.

Palabras Clave: autoconcepto, rendimiento, estudiantes universitarios, regresión logística, curva ROC.

\section{ABSTRACT}

Academic performance represents a multidimensional phenomenon; so it is possible to tackle it considering many different factors, its links and the implications it may have. In this context, the main aim of this study is to develop a logistic regression model that allows to explain in which ways the different areas of self-concept construct is connected to the academic performance results. The sample, formed by students belonging to a national public university (state funded) of Argentina, was chosen in a stratified manner, by conglomerates and randomly. It is composed of 164 young people ( 91 women and $73 \mathrm{men}$ ) with a median age of 19.80 years $(S D=1.72)$. The research is accountable to an explanatory and descriptive design through a quantitative and cross-sectional study (self-concept test form 5: academic, social, emotional, 
family and physical). During the empiric phase, statistical, descriptive, psychometric and inferential analyses allowed us to learn certain characteristics of the dimensions of the test, the internal consistency indices from the different areas and the instrument as a whole; thus, it has allowed us to determine the most appropriate logistic model that best adjusts to sample data. The concept of ROC curve has been employed in order to show the global capacity the model has to explain the results of academic performance. To sum up, it can be said that the survey used is a reliable test that has a predictive capacity to describe the variability of the academic results on the basis of the different types of self-concept analyzed.

Keywords: university students, self-concept, performance, logistic regression, ROC curve.

\section{1.- INTRODUCCIÓN}

El rendimiento académico constituye la manifestación formal y expresa del grado de aprendizaje del alumno, y se infiere tradicionalmente a partir de sus calificaciones. Es sabido que representa un fenómeno multidimensional, que es posible abordarlo tomando en cuenta distintas variables, sus relaciones y las implicancias que en él pueden tener.

En el ámbito de países que poseen un régimen público de educación superior, como lo es Argentina, los resultados educativos constituyen una preocupación de sumo interés. Esto se debe a que el objetivo final de la provisión gratuita de este nivel de enseñanza es la igualación de oportunidades y el ascenso social de los ciudadanos, lo cual depende directamente del éxito que tengan los alumnos universitarios en términos del aprovechamiento y finalización de sus estudios.

Para la Universidad Nacional del Nordeste (UNNE), que forma parte del Sistema Universitario Argentino y en cuyo ámbito se lleva a cabo el presente estudio, el tema del bajo rendimiento académico es aún de mayor relevancia social. Esto es así en razón de que su zona de influencia incluye una de las áreas con mayor nivel de subdesarrollo educativo y económico, como lo es la región nordeste.

El insuficiente rendimiento académico, que se puede explicar por inconvenientes de distinta índole y origen, arrastra a muchos alumnos al abandono de sus estudios -lo que implica un costo social considerable-, siendo una de las causas más frecuente la imposibilidad de aprobar algunas de las asignaturas de primer año.

Si bien existen múltiples factores que inciden en el rendimiento académico, sólo nos enfocamos aquí en el autoconcepto de los estudiantes, como aquella variable motivacional en la que, de acuerdo con algunos autores (Núñez et al., 1998), se pone de manifiesto que la implicación activa del sujeto en su proceso de aprendizaje se incrementa cuando se percibe autoeficiente. 
Como suele ocurrir con muchos otros términos, existen diferentes significados, conceptualizaciones y descripciones relacionadas con el constructo bajo estudio. Así, en el diccionario de la Real Academia Española (2014), la definición sobre autoconcepto indica que es la "opinión que una persona tiene sobre sí misma, que lleva asociado un juicio de valor".

A su vez, de acuerdo con el diccionario Akal de Psicología (Doron y Parot, 1998), la noción de autoconcepto consiste en una "representación de sí en el sistema de conocimientos del individuo. Esta representación es equivalente a una estructura cognitiva probablemente compleja que interviene en el tratamiento de las informaciones procedentes del entorno social del individuo o de su propio comportamiento" (p. 69).

Diversos autores se han ocupado del vocablo y de la problemática objeto de este trabajo. Así por ejemplo, Burns (1982) define el autoconcepto como la percepción que el sujeto tiene de sí mismo; está basado en las experiencias individuales y sociales y en las atribuciones que se otorgan a la propia conducta; incluye actitudes, sentimientos, apariencias, aceptación social y capacidades cognitivas. Es considerada como una de las variables personales que mayor influencia tendría, tanto directa como indirectamente, en el rendimiento académico.

El interés por el autoconcepto, ha estado presente desde hace tiempo en el psicoanálisis, el conductismo, las teorías del aprendizaje social, la psicología cognitiva y la psicología humanística; también en el campo de la psicología aplicada: clínica, educativa y social (Harter, 1986).

En la década del sesenta, los modelos del autoconcepto eran típicamente de naturaleza unidimensional, es decir, consideraba que el autoconcepto era un constructo unitario que podía ser evaluado presentando a niños o adolescentes ítems que reflejaran su autoconcepto global a través de múltiples contextos. Hacia los años ochenta, en cambio, las investigaciones abandonaron este enfoque unidimensional y desarrollaron un modelo multidimensional propuesto por Shavelson, Hubner y Stanton (1976).

En este modelo se distinguen un autoconcepto general que se subdivide en académico (Inglés, Historia, Matemáticas y Ciencias) y no académico (estados emocionales particulares) y físico (habilidad y apariencia física). Este modelo propone que el autoconcepto pueda ser evaluado utilizando instrumentos que midan cada una de las áreas por separado (Escurra, 2005).

Según sostiene Marsh (1993), se entiende por autoconcepto académico, a la concepción que tiene el estudiante de su capacidad para aprender y rendir en las tareas escolares. La bibliografía científica lo valora como una condición necesaria pero no suficiente para un adecuado rendimiento. Este autoconcepto académico, incluye al autoconcepto de áreas (Ciencias Biológicas, Matemáticas, Ciencias Sociales, Lenguaje) que constituye la percepción que el estudiante tiene sobre las posibilidades de obtener un relativo éxito en los contenidos de cada una de ellas.

Respecto del autoconcepto no académico, Shavelson, Hubner y Stanton (1976) expresan que se configura por componentes emocionales -son los más subjetivos e internos-, sociales -relacionados con el significado que la conducta del individuo tiene para los demás-y físicos -en los que tienen una incidencia fundamental las actitudes y apariencia general del individuo-. 
En el presente trabajo se analizó el autoconcepto de los alumnos a partir del test Autoconcepto Forma 5 (AF5), elaborado por García y Musitu (2009), el que se encuentra conformado por treinta (30) afirmaciones, organizadas en seis (6) ítems para cada una de las cinco (5) áreas consideradas: académica, social, emocional, familiar y física, las cuales brevemente pasamos a describir.

Académica: se refiere a la percepción que el sujeto tiene de la calidad del desempeño de su rol como estudiante. Social: es la opinión que tiene el individuo de su desempeño en las relaciones sociales. Emocional: hace referencia a la apreciación que una persona realiza respecto de su estado emocional y de sus respuestas a situaciones específicas, con cierto grado de compromiso e implicación en su vida cotidiana. Familiar: está asociado a la consideración que tiene el sujeto de su implicación, participación e integración en el medio familiar. Física: este factor se vincula con la creencia que tiene el individuo de su aspecto físico y de su condición física.

Los estudios sobre autoconcepto han demostrado que este constructo constituye uno de los más importantes y significativos reguladores de la conducta humana (Suls, 1982; Suls y Greenwald, 1983). No obstante, hay dificultades para establecer la naturaleza de la relación y para identificarla. De acuerdo con Markus y Wurf (1987) el inconveniente con el que nos encontramos en el momento de identificar la influencia del factor en la conducta del individuo radica también, en estimar qué otros aspectos influyen en la conducta, además del autoconcepto.

En particular, respecto a la relación causal entre el autoconcepto y el rendimiento académico, los resultados de investigaciones realizadas no aportan evidencia definitiva sobre la naturaleza exacta de la dirección del vínculo que une a estas dos variables. En efecto, Núñez y González (1994) distinguen cuatro patrones o modelos de causalidad entre ambos constructos. En primer lugar, el rendimiento académico como determinante del autoconcepto; en segundo término, los niveles del autoconcepto como determinantes del grado de logro académico; en tercer orden, autoconcepto y rendimiento académico se influyen y determinan mutuamente; por último, terceras variables pueden ser la causa tanto del autoconcepto como del rendimiento académico.

Con el fin de explicar de la mejor forma posible -tanto conceptual como técnica- de qué manera el constructo psicológico objeto de interés influye en los resultados académicos, nos hemos planteado como objetivo en este estudio desarrollar un modelo de regresión logística multivariable. En la fase empírica de esta investigación, la variable dependiente o explicada de la ecuación de regresión será, ciertamente, el rendimiento académico (medido a través de las calificaciones parciales obtenidas en la asignatura de primer año, Contabilidad Básica [CB], la cual es común para las distintas carreras que se imparten en la Facultad de Ciencias Económicas [FCE] de la UNNE), mientras que las variables predictoras o explicativas serán las diferentes dimensiones del factor autoconcepto (evaluadas mediantes la prueba $\mathrm{AF}_{5}$ ) propuestas por García y Musitu (2009). 


\section{2.- MÉTODO}

\subsection{PARTICIPANTES}

Debido a que nuestro interés radica en trabajar con una muestra en la cual su unidad se encuentre formada por la totalidad de los estudiantes que componen una entidad con definida personalidad como es el grupo-clase, hemos considerado adecuado optar por dos regímenes de promoción: a) pruebas parciales y final oral (PPFO), y b) pruebas parciales acumulativas (PPA); los cuales se ofrecen, a su vez, bajo dos modalidades de cursado: a) presencial, y b) semipresencial, todo ello en el marco de la asignatura CB de la FCE-UNNE.

Cabe señalar que bajo el régimen de promoción PPA, la modalidad de cursado es tanto presencial como semipresencial, mientras que en el régimen por PPFO, la modalidad es únicamente presencial. En razón de lo señalado, se puede sostener que la muestra ha sido seleccionada utilizando los métodos estratificado (las opciones de cursado representaban los estratos) y por conglomerados (los grupos-clase o comisiones de estudio integraban los cluster), los cuales fueron seleccionados de manera aleatoria.

Concretamente, la muestra estuvo compuesta por 6 grupos-clase ( 2 para el régimen de PPFO, 2 para el régimen de PPA presencial y 2 para el régimen de PPA semipresencial), los que totalizaban 164 jóvenes, 91 mujeres (55.49\%) y 73 hombres (44.51\%), con una media de 19.81 años y desviación estándar de 1.72. Algunas de las característitcas de la muestra utilizada en esta investigación, se ilustran en la Tabla 1.

Tabla 1. Detalles relativos a la muestra empleada en la investigación empírica

\begin{tabular}{|c|c|c|c|}
\hline Modalidad & Asignatura & Alumnos & Edad \\
\hline PPFO & \multirow{3}{*}{ Contabilidad Básica } & $n=58(31 \mathrm{~m}, 27 \mathrm{~h})$ & $\begin{array}{l}\text { Mín. }=18 \quad \text { Máx. }=25 \\
M=19.47 \quad D E=1.61\end{array}$ \\
\hline $\begin{array}{c}\text { PPA } \\
\text { presencial }\end{array}$ & & $n=30(17 \mathrm{~m}, 13 \mathrm{~h})$ & $\begin{array}{l}\text { Mín. }=18 \quad \text { Máx. }=25 \\
M=20.80 \quad D E=1.65\end{array}$ \\
\hline $\begin{array}{c}\text { PPA } \\
\text { semipresencial }\end{array}$ & & $n=76(43 \mathrm{~m}, 33 \mathrm{~h})$ & $\begin{array}{l}\text { Mín. }=18 \quad \text { Máx. }=25 \\
M=19.67 \quad D E=1.70\end{array}$ \\
\hline \multicolumn{4}{|c|}{ Muestra: $N=164(91 \mathrm{~m}, 73$ h) Edad: Mín. $=18$, Máx. $=25, M=19.81, D E=1.72$} \\
\hline
\end{tabular}

Fuente: Elaboración propia

\subsection{DISEÑO}

Esta investigación, inicialmente de naturaleza no experimental, puede considerarse en un segundo momento también explicativa, debido al objetivo que persigue. Si consideramos como criterio el tipo de información que se proveerá y el modo de reunirla, el diseño es de estilo descriptivo mediante encuesta.

Por otra parte, en atención a la forma de administrar el instrumento de medición, en este estudio empleamos la técnica del cuestionario. A su vez, si tenemos en cuenta el marco donde 
se lleva a cabo, estaríamos hablando de una investigación de campo. Además, en razón de cómo se miden y analizan los datos, es una investigación de línea cuantitativa. Teniendo en cuenta la instancia de recolección de la información, este trabajo revela una estrategia de corte transversal. En virtud del interés por analizar las asociaciones entre las distintas variables que participan, el presente estudio es de perfil correlacional.

En líneas generales, desde el ámbito de la confrontación teórica-empírica, podríamos señalar que se trata de un proceso de carácter hipotético-deductivo, puesto que pretendemos comprobar si la conceptualización teórica de la cual partimos se ajusta a la realidad objeto de estudio, a través de la recogida de datos, su posterior análisis estadístico y la interpretación de los resultados obtenidos.

\subsection{PROCEDIMIENTO}

Una vez elegida la muestra, la recolección de los datos se realizó, en cada uno de los grupos-clase, en una única instancia. En primer lugar se les informó a los alumnos participantes que la aplicación del instrumento en cuestión (test AF5) respondía a un trabajo de investigación relacionado con temas de rendimiento académico, que tiene la intención -a partir de la lectura e interpretación de sus resultados- de aportar propuestas de intervención que posibiliten mejorar el fenómeno objeto de estudio. También se les indicó sobre la importancia de responder sinceramente a los distintos ítems planteados, que sus respuestas tendrían un carácter estrictamente confidencial, sólo de uso científico y que la participación en el estudio era una decisión totalmente voluntaria.

El momento temporal de este proceso fueron los meses de septiembre y octubre de 2016. La aplicación del cuestionario la efectuaron los propios profesores, al comienzo de clase y con el margen de tiempo adecuado en virtud de las consultas formuladas en la prueba (20 minutos en promedio). Concluido el trabajo de campo propiamente dicho y el ordenamiento de la información obtenida, se procedió a la construcción de la matriz de datos en formato electrónico, así como a su posterior control general.

\subsection{INSTRUMENTOS}

Para cumplir con el objetivo propuesto y recoger los datos relativos al tema bajo estudio se utilizó, según fuera anticipado, el test AF5 cuya autoría pertenece a García y Musitu (2009). Esta prueba se encuentra compuesta por 30 afirmaciones agrupadas en 5 dimensiones (6 ítem en cada área): académica (p. ej., Soy un buen estudiante), social (p. ej., Hago fácilmente amigos), emocional (p. ej., Muchas cosas me ponen nervioso), familiar (p. ej., Mis padres me dan confianza) y física (p. ej., Soy bueno haciendo deportes), acerca de las que nos hemos referido brevemente en el apartado introductorio. 
Para responder a cada una de las afirmaciones planteadas en el instrumento utilizado los estudiantes disponían de una escala con alternativas que iban de 1 a 99. La aplicación del test podía realizarse en forma individual o colectiva, en nuestro caso evidentemente se realizó en forma colectiva. En virtud de lo que antecede, se trabajó con fuentes de información primaria; esto es, se aplicó la prueba mencionada y se recogieron los datos. Para esta acción, ciertamente, el investigador asumió el rol de observador y los alumnos el carácter de informantes.

A su vez, con la finalidad de obtener el modelo logístico que mejor permita explicar o predecir la varianza de los resultados académicos, así como contrastar la validez predictiva del instrumento, hemos empleado como variable respuesta las calificaciones (promedio de evaluaciones parciales) alcanzadas por los alumnos encuestados en la asignatura CB, las que fueron obtenidas a partir de las actas académicas (fuentes de datos secundarios), disponibles en el Sistema de Gestión Universitaria SIU-Guaraní.

Se han seleccionado las calificaciones, puesto que son el criterio social y legal del rendimiento en el ámbito de los centros educativos, además de ser el indicador más utilizado en las investigaciones sobre esta temática. La variable dependiente (calificaciones) del modelo es de tipo continua, sus valores enteros varían entre 1 y 10; en cambio, las variables independientes (dimensiones de la $\mathrm{AF}_{5}$ ), si bien son continuas, sus valoraciones oscilan entre 0.10 y 9.90.

\subsection{ANÁLISIS DE DATOS}

En primer término, debemos señalar que la revisión cualitativa del instrumento AF5, fue efectuada por un grupo de profesores de los Departamentos de Matemática y de Contabilidad de la FCE-UNNE, en cuanto a dos aspectos: a) pertinencia del contenido de los ítems propuestos (indicadores subjetivos de validez), y b) conformación del cuestionario en su conjunto (indicadores de la validez factorial o estructural), habiendo sido favorable en ambos casos.

Los análisis realizados en la línea de validez cualitativa (juicio de expertos y grado de acuerdo) resultaron verdaderamente valiosos, puesto que permitieron minimizar los márgenes de error del test al momento de su utilización en nuestro espacio académico. La validez de una prueba es un indicador del grado en que ésta es capaz de medir lo que realmente pretende evaluar, por lo que resulta relevante inicialmente su valoración cualitativa, así como su evaluación cuantitativa en la etapa empírica de la investigación.

Ahora sí, luego de construida la base de datos en formato electrónico a partir de la información obtenida por la aplicación del test, y de las calificaciones de los alumnos de la muestra, se llevaron a cabo diversos análisis estadísticos. Los estudios implementados en principio pertenecen al dominio de la estadística descriptiva (algunos estadísticos centrales 
y de dispersión), así como al ámbito de la psicometría (correlación dimensión-total corregida y consistencia interna). En cambio, los cálculos desarrollados posteriormente se encuentran en el área de la estadística inferencial (análisis correlaciónales bivariados, análisis de regresión logística y curva ROC; para las pruebas de hipótesis, como es habitual, utilizamos la medida $p$-valor).

Los diferentes tratamientos estadísticos indicados en el párrafo anterior permitieron, por un lado, conocer las características y el comportamiento de cada una de las áreas de la prueba utilizada, así como el grado de confiabilidad del instrumento; por otra parte, dieron lugar a determinar la ecuación de predicción que mejor describía la relación entre los cinco tipos de autoconcepto considerados (variables explicativas o predictoras) y el rendimiento académico (variable explicada o respuesta). En todos los casos, el procesamiento de los datos fue realizado con ayuda del programa IBM SPSS Statistics 22.

\section{3.- RESULTADOS}

\subsection{ESTUDIOS DE LAS DIMENSIONES DEL TEST APLICADO}

Los análisis estadísticos que estarían en condiciones de ser realizados en el marco de esta investigación, a partir de los datos disponibles, podrían ser numerosos; sin embargo, como se comprenderá, sólo se llevarán a cabo aquellos cálculos que verdaderamente respondan a los estudios mencionados en el apartado anterior. En virtud de ello, se presentan a continuación de forma sintética los resultados relativos a los indicadores descriptivos y psicométricos que nos han parecido más convenientes para caracterizar la muestra en las diferentes dimensiones de la prueba aplicada.

En efecto, en la Tabla 2 pueden apreciarse las valoraciones (mínimas y máximas), la media, la desviación estándar, la correlación dimensión-total corregida y el coeficiente alfa de Cronbach.

Los tres primeros estadísticos son de mucha utilidad, puesto que cuando se analiza un conjunto de datos numéricos, el conocimiento de dichas medidas ayuda a comprender, entre otras cosas, la distribución de los datos de la muestra.

El cuarto de los cinco estadísticos mencionados (correlación dimensión-total corregida), recoge el grado de relación que cada una de las áreas posee con el total de la prueba, lo que puede considerarse un indicador de su grado de discriminación.

La fiabilidad, una de las características fundamentales de un test, si bien puede ser evaluada de diferentes maneras, en esta investigación se realizó a través del quinto estadístico (coeficiente alfa de Cronbach), este indicador revela la precisión o estabilidad de los resultados; señala la cuantía en que las medidas de la prueba están libres de errores casuales o aleatorios. 


\begin{tabular}{c}
\hline ANTONIO H. CLOSAS - NOELIA B. FRANCHINI - LUCIANA CYNTHIA KUC - MARÍA ALICIA DUSICKA - CARLOS M. HISGEN \\
MODELO LOGISTICO EXPLICATIVO DE LAS RELACIONES ENTRE AUTOCONCEPTO Y RENDIMIENTO ACADÉMICO \\
Págs. 187 - 208. DOI: $h$ ttp: $/ / d x$.doi.org/10.30972/rfce.0203261
\end{tabular}

Tabla 2. Estadísticos descriptivos, de correlación y de fiabilidad de las dimensiones de la AF5

\begin{tabular}{|c|c|c|c|c|c|c|}
\hline Dimensión & $\begin{array}{c}\text { Número } \\
\text { de ítems }\end{array}$ & Valoración & Media & $D E$ & $\begin{array}{c}\text { Correlación } \\
\text { dimensión-total } \\
\text { corregida }\end{array}$ & $\begin{array}{c}\alpha \text { de Cronbach } \\
\text { sin la dimensión }\end{array}$ \\
\hline Académica & 6 & $\begin{array}{c}\text { Mín. }=1.20 \\
\text { Máx. }=9.25\end{array}$ & 5.92 & 1.61 & .42 & .68 \\
\hline Social & 6 & $\begin{array}{c}\text { Mín. }=1.92 \\
\text { Máx. }=9.90\end{array}$ & 6.53 & 1.89 & .59 & .59 \\
\hline Emocional & 6 & $\begin{array}{c}\text { Mín. }=0.10 \\
\text { Máx. }=9.77\end{array}$ & 5.21 & 2.14 & .41 & .69 \\
\hline Familiar & 6 & $\begin{array}{c}\text { Mín. }=2.67 \\
\text { Máx. }=9.90\end{array}$ & 8.26 & 1.65 & .35 & .71 \\
\hline Física & 6 & $\begin{array}{c}\text { Mín. }=0.38 \\
\text { Máx. }=9.57\end{array}$ & 5.91 & 1.91 & .59 & .59 \\
\hline AF5 (5 Dimensiones): Val. Mín. $=13.59$ & Val. Máx. $=44.86$ & Media $=31.83$ & $D E=5.78 \quad \alpha=.71$ \\
\hline
\end{tabular}

Fuente: Elaboración propia

A continuación se destacan algunos aspectos que surgen de la lectura de los valores que se encuentran en la Tabla 2, obtenidos por cierto a partir de los análisis efectuados sobre los datos muestrales.

Comenzamos por señalar que los valores hallados para cada una de las dimensiones, así como para el conjunto de las mismas, en cuanto a valoración, media y desviación estándar, resultaron razonables y se encuentran dentro del rango de medidas que se esperaban obtener, en virtud de los antecedentes bibliográficos que fueron consultados sobre el tema, y de que no se realizaron modificaciones de ningún tipo en el texto de las preguntas, ni en la estructura de la prueba original.

En general, las valoraciones en cada una de las categorías muestran correlaciones corregidas aceptables con las valoraciones totales en la prueba (sumatoria de los ítems que componen las dimensiones, excluidos aquellos que integran la dimensión cuya asociación se desea evaluar), puesto que en todos los casos superan sin inconvenientes el valor de referencia .20 (Kline, 2000), observándose las más altas en las áreas denominadas Social (.59) y Física (.59).

Respecto de los indicadores a de Cronbach cuando se excluye la dimensión, podemos señalar que las cifras destacadas corresponden a las áreas Social (.59) y Física (.59), citadas anteriormente (en esta ocasión, al contrario de lo que sería conveniente en el contexto de las correlaciones corregidas, coeficientes bajos ponen en evidencia el aporte relevante que la dimensión que no participa realiza respecto de la fiabilidad de la prueba). Las medidas $\alpha$ de Cronbach halladas pueden considerarse correctas en general, ya que tres de ellas satisfacen, y las dos restante están muy próximas de hacerlo, el criterio de algunos autores de valer en estudios exploratorios al menos .6o (Huth, Delorme y Reid, 2006).

Los indicadores de las dos últimas columnas de la Tabla 2 pertenecen a conceptos estrechamente vinculados, en términos generales, con la confiabilidad del instrumento, que en nuestro estudio resultaron muy coherentes y sencillos de interpretar (valores altos de corre- 
laciones corregidas se relacionan con cuantificaciones bajas de $\alpha$ de Cronbach, que esta oportunidad casualmente resultaron idénticos, cuando se consideran sólo dos dígitos decimales).

Para finalizar con este apartado, debemos señalar que la fiabilidad calculada para el conjunto de las cinco dimensiones es aceptable puesto que el coeficiente alfa encontrado (.71) supera el criterio de .70 recomendado (Nunnaly y Bernstein, 1994). Se considera conveniente mencionar que calcular el coeficiente de fiabilidad en cada nueva muestra, y no apoyarse en la obtenida en otros estudios como aval de la fiabilidad del instrumento, es una de las recomendaciones de la American Psychological Association (2009), como también de la política editorial de importantes revistas (Thompson, 1994).

\subsection{ANÁLISIS CORRELACIÓNALES BIVARIADOS}

En este apartado se describirán los resultados de los análisis relacionales entre las cinco dimensiones que integran el test $\mathrm{AF}_{5}$ y la variable rendimiento académico (los datos de esta variable, originalmente oscilaban entre 1 y 10 , fueron recodificados: a las calificaciones entre 1 y 5 se les asignó el valor 1, mientras que a las notas comprendidas entre 6 y 10 les correspondió el valor o).

La primera razón por la que se realizan estos estudios radica en el hecho de que los estadísticos que se obtengan permitirán reconocer la presencia o no de asociaciones entre las categorías del instrumento y los resultados académicos, lo que proporcionará un indicio acerca de la validez predictiva de la prueba objeto de interés.

El segundo motivo de los actuales estudios reside en que, en atención al objetivo principal de esta investigación, está previsto modelizar mediante regresión logística las relaciones entre las dimensiones del cuestionario aplicado y el rendimiento académico, y es siempre de utilidad examinar previamente las asociaciones que presentan, en esta ocasión, las variables independientes con la variable dependiente del futuro modelo.

Respecto de los valores del estadístico $t$ de Student (permite contrastar la hipótesis nula de que el rendimiento es independiente del autoconcepto) entre las dimensiones de la prueba AF5 y el rendimiento académico, según puede verse en la Tabla 3, de los cinco posibles, sólo uno resultó estadísticamente significativo $(\alpha=.01)$, tal es el caso del correspondiente al autoconcepto Académico $(t=-4.86)$. El signo negativo obtenido en el resultado del test se debe a que la variable dicotómica ha sido codificada en sentido contrario a las valoraciones de la variable continua.

Tabla 3. Relaciones entre las dimensiones de la prueba AF5 y el rendimiento académico

\begin{tabular}{|l|c|c|c|c|c|}
\hline & Académica & Social & Emocional & Familiar & Física \\
\hline Rendimiento académico & $-4.86^{* *}$ & .10 & -.86 & -.27 & .22 \\
\hline
\end{tabular}

${ }^{* *} p<.01 \quad N=164$

Nota: Para determinar si las distintas áreas del test (variables continuas) se hallaban relacionadas con el rendimiento académico (variable dicotómica), se realizaron contrastes de hipótesis a través de la prueba t de Student. Fuente: Elaboración propia 
Lo destacable de los indicadores obtenidos en esta parte del estudio es que la presunción que teníamos al respecto; esto es, la presencia de asociación entre ambos constructos (autoconcepto y rendimiento), pudo ser empíricamente comprobada. Más precisamente podemos afirmar que en este contexto de análisis estadísticos, se encontraron evidencias suficientes para rechazar la hipótesis nula de que el rendimiento académico no depende del autoconcepto Académico; de manera que es posible señalar por ahora, que al menos la dimensión mencionada se encuentra efectivamente relacionada con la variable de respuesta.

Esta apreciación nos lleva a sostener, a priori, que principalmente la categoría Académica, por evidentes razones, podría ser de utilidad para configurar un modelo que permita clasificar en el futuro el desempeños de los estudiantes encuestados; aunque de ninguna manera deberían descartarse las demás dimensiones como posibles variables independientes de la ecuación final de regresión.

\subsection{REGRESIÓN LOGÍSTICA BINARIA}

En vista del objetivo planteado en este estudio, ha sido ingresada como variable explicada o de respuesta el Rendimiento académico ( $\mathrm{o}=$ Aprobado y 1 = Desaprobado), y como variables explicativas o covariables las cinco dimensiones del test AF5: Académica, Social, Emocional, Familiar y Física.

Sin embargo, los resultados de la regresión logística binaria (en SPSS optamos por el método Atrás: Condicional) indican, en virtud de la aplicación de los test de ajuste global, que las variables Académica, Social, Emocional y Física, en su conjunto, serían relevantes a la hora de explicar o predecir el comportamiento de los resultados académicos en la asignatura CB. La única área del cuestionario empleado en el estudio que ha sido excluida del modelo fue el autoconcepto Familiar; la razón de ello se debió a que sus indicadores lo sugerían de manera categórica, y porque además los estadísticos de bondad de ajuste del modelo se veían favorecidos.

En concreto, respecto al contraste global del modelo (véase Tabla 3), podemos indicar que el p-valor correspondiente a la prueba Chi-cuadrado (24.95) ha resultado .oo; por lo que, para un nivel de significación $\alpha=.05$, se rechaza la hipótesis nula de que los coeficientes incluidos en el modelo sean estadísticamente iguales a cero.

A su vez, la prueba de Hosmer-Lemeshow (la hipótesis nula indica que el modelo se ajusta a la realidad), otra forma de evaluar la bondad de ajuste de un modelo de regresión logística, ha proporcionado un p-valor de .59, para el estadístico Chi-cuadrado cuya medida resultó 6.48 (Tabla 3); de manera que en sintonía con lo expresado en el párrafo anterior, podemos sostener que el modelo que se propone refleja adecuadamente los datos empíricos (no se encontraron evidencias suficientes para rechazar la hipótesis nula). 
Tabla 3. Indicadores globales del modelo

\begin{tabular}{|c|c|c|}
\hline Test & $\boldsymbol{\chi}^{\mathbf{2}}$ & Valor $\boldsymbol{p}$ \\
\hline Bondad de ajuste & 24.95 & .00 \\
\hline Hosmer-Lemeshow & 6.48 & .59 \\
\hline
\end{tabular}

Fuente: Elaboración propia

Los coeficientes $B$ del modelo, los estadísticos de Wald, y los respectivos p-valores, pueden apreciarse en la Tabla 4. Según se puede observar, con excepción de la categoría Académica, las demás dimensiones de la prueba AF5: Social, Emocional y Física, poseen valores bajos en los coeficientes de regresión y, por cierto, indicadores de contraste con grados de significación estadística asociada superiores a .05. En razón de ello, a la hora de seleccionar el modelo que razonablemente se ajusta a los datos de la muestra, se han priorizado en general los resultados de los test de ajuste global, por encima de criterios particulares (básicamente, prueba de Wald y p-valor) respecto de cada una de las tres últimas áreas mencionadas.

Tabla 4. Coeficientes del modelo y estadísticos de Wald

\begin{tabular}{|c|c|c|c|}
\hline & $\boldsymbol{B}$ & Wald & Valor $\boldsymbol{p}$ \\
\hline Académica & -.57 & 19.40 & .00 \\
\hline Social & .09 & .69 & .40 \\
\hline Emocional & -.06 & .45 & .50 \\
\hline Física & .12 & 1.35 & .24 \\
\hline Constante & 2.52 & 8.03 & .01 \\
\hline
\end{tabular}

Fuente: Elaboración propia

De acuerdo con Jovell (1995), los propósitos del modelo logístico residen en determinar la existencia o ausencia de relación entre una o más variables independientes y la variable dependiente; medir la magnitud de dicha relación y explicar y/o predecir la probabilidad de que la variable respuesta sea igual a 1, en función de los valores que adopten las variables predictoras.

Así pues, en virtud de todo lo que antecede, se procedió a plantear un modelo de regresión logística conformado por cuatro de las cinco dimensiones posibles como variables explicativas de la ecuación, el resultado obtenido pueden apreciarse a continuación:

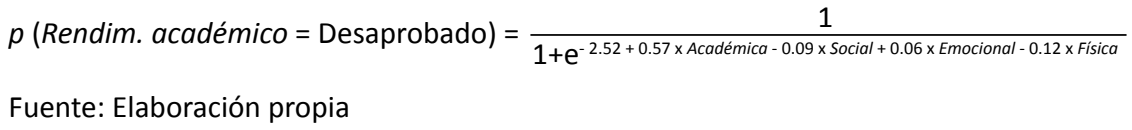

Si bien en este apartado hemos sostenido que el modelo propuesto se ajusta a los datos de la muestra, utilizaremos a continuación el concepto de la curva ROC (Receiver Operating Characteristic), con el objeto de mostrar la capacidad que el modelo posee para explicar los resultados del rendimiento académico, así como de elegir el punto de corte más apropiado 
para una sensibilidad o una especificidad determinada. La sensibilidad indica la capacidad del estimador para identificar correctamente los casos positivos (en nuestro estudio, alumnos que se encuentran en el grupo de desaprobados; es decir, estudiantes que presentan problemas de rendimiento). Por el contrario, la especificidad es la probabilidad de detectar correctamente la presencia de casos negativos (en nuestro estudio, alumnos que se encuentran en el grupo de aprobados, o que carecen de dificultades académicas).

\subsection{CURVA ROC}

En la Tabla 5 se presentan diferentes valores del área bajo la curva ROC. En efecto, pueden apreciarse la estimación puntual (.72), el error estándar de esta estimación (.04), también el límite inferior (.64) y superior (.79) de un intervalo de confianza del 95\%. Como este intervalo no contiene al valor .50, podemos rechazar la hipótesis nula (AUC [Área Under the Curve] = .50) y concluir que la estimación puntual del área bajo la curva $\operatorname{ROC}(.72, p<.05)$ estaría indicando que el modelo que se propone posee calidad diagnóstica para clasificar el Rendimiento académico de los estudiantes de la muestra.

De la observación de la lista de coordenadas de la curva ROC (información obtenida a partir de las alternativas seleccionadas y las opciones que por defecto brinda SPSS 22), surge que para el caso de una sensibilidad del $76 \%$ tendríamos una especificidad del $64 \%$, lo que se consigue en el punto de corte 0.48 .

El punto de corte lo hemos elegido teniendo en cuenta que la sensibilidad fuera la más alta y el número de falsos positivos (1 - especificidad) fuera el más bajo, dentro de los valores posibles, puesto que de esta manera, además de maximizar el índice de Yuoden, el modelo proporcionará estimaciones que estarían equilibradas y ajustadas a la realidad objeto de estudio.

Tabla 5. Área bajo la curva ROC

\begin{tabular}{|c|c|c|c|c|}
\hline \multirow{2}{*}{ Área } & \multirow{2}{*}{ Error estándar } & \multirow{2}{*}{ Valor $p$} & \multicolumn{2}{|c|}{ Intervalo de confianza del 95\% } \\
\cline { 4 - 5 } & & & Límite inferior & Límite superior \\
\hline .72 & .04 & .00 & .64 & .79 \\
\hline
\end{tabular}

Fuente: Elaboración propia

En la Figura 1, puede apreciarse la representación gráfica de la curva ROC ajustada a los datos muestrales. Se observa que la curva se encuentra razonablemente por encima de la recta $y=x$, por lo que podemos considerar que el método de diagnóstico es aceptable para discriminar los resultados educativos. La flecha indica el punto de corte óptimo (o.48) que determina la sensibilidad (0.76) y especificidad $(1-0.36=0.64)$ conjunta más alta (Mayor índice de Youden $=$ Sensibilidad + Especificidad $-1=0.40)$. 


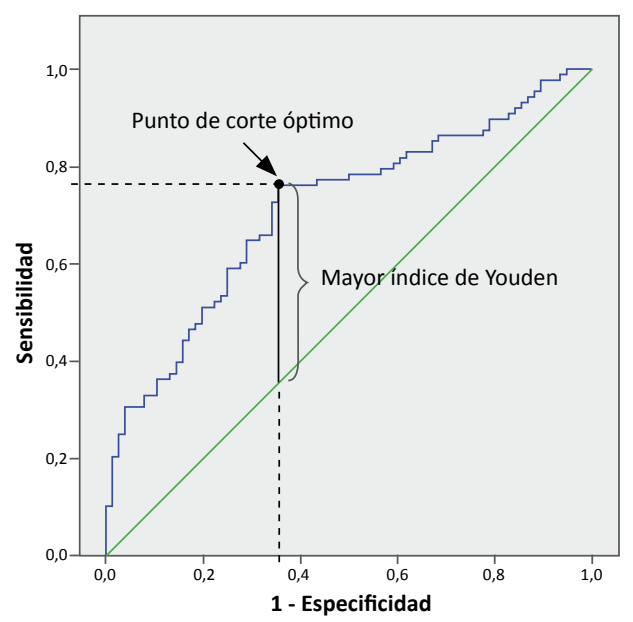

Figura 1. Gráfico de la curva ROC

Fuente: Elaboración propia

Como se podrá comprender, el punto de corte óptimo no necesariamente determina la sensibilidad ni la especificidad más alta que podría alcanzar el test (generalmente la sensibilidad más alta es determinada por un punto de corte, mientras que la especificidad más alta es determinada por otro).

En efecto, existen situaciones en las que se requiere disponer de un test diagnóstico altamente sensible (p. ej., para identificar estudiantes que podrían presentar o que revelan dificultades en el desempeño) o bien altamente específico (p. ej., para reconocer alumnos que no tendrían o que no poseen problemas de rendimiento). En tales circunstancias, no es aconsejable utilizar el punto de corte identificado por el mayor índice de Youden; por el contrario, resulta más útil conocer los valores de sensibilidad y especificidad determinados por diferentes puntos de corte, y optar por aquél que determine la sensibilidad, o la especificidad, que se desee en virtud del objetivo propuesto.

Así por ejemplo, en el contexto de este estudio, para valores de sensibilidad $=0.81 \mathrm{y}$ de especificidad $=0.41$, el punto de corte deberá ser: 0.40 (Índice de Youden $=0.22$ ); esto es, inferior al óptimo (0.48) vinculado anteriormente con los máximos valores conjuntos de sensibilidad (0.76) y especificidad (0.64).

Por el contrario, para valores de sensibilidad $=0.71 \mathrm{y}$ de especificidad $=0.66$, el punto de corte deberá ser: 0.50 (Índice de Youden $=0.37$ ), levemente superior al óptimo que se asocia con la mayor sensibilidad y especificidad conjunta.

Obsérvese que el índice de Youden en los dos ejemplos precedentes, como corresponde, resultó inferior a 0.40 ; esto es, al valor máximo del mencionado indicador. 
Todas estas apreciaciones se realizan en el marco de las posibilidades acotadas que el modelo posee para explicar y predecir la varianza del rendimiento de los individuos de la muestra, a partir de las valoraciones en las dimensiones que participan. Por lo tanto, las estimaciones e interpretaciones efectuadas deben ser consideradas con debida prudencia, en razón del particular escenario empírico en el que se ha desarrollado el trabajo y de la metodología de abordaje que ha sido implementada, circunstancias ambas que indudablemente limitan las bondades que la presente investigación pudiera ofrecer.

Sin embargo, en atención a que el número de aciertos globales del modelo es uno de los indicadores más importante de la bondad de ajuste del mismo, podemos sostener que la ecuación estimada resulta razonable para clasificar y predecir las categorías de la variable dependiente. En efecto, lo anterior se apoya en el hecho de que si se aplicara el modelo propuesto a las observaciones muestrales, se obtendría un porcentaje de éxitos que estaría comprendido en el 64\% y 79\%, con un nivel de confianza del 95\% (ver Tabla 5).

En definitiva, y a modo de síntesis, podría expresarse que la investigación puesta en marcha nos ha permitido contrastar empíricamente, mediante la utilización de la ecuación de regresión, que aquellos estudiantes que se caracterizan por tener valoraciones iguales o superiores a la media en las áreas Académica (5.92) y Emocional (5.21), sin necesidad que suceda los mismo con las otras dos dimensiones que forman parte del modelo Social (6.53) y Física (5.91), sino más bien lo contrario, tendrían mayores probabilidades (en el modelo logit los valores de la variable dependiente estarían por debajo del punto de corte óptimo) de alcanzar mejores resultados en la asignatura CB.

Por cierto, sucedería lo contrario; esto es, bajas probabilidades de alcanzar los objetivos del curso (en la ecuación de regresión los valores de la variable explicada estarían por encima del punto de corte óptimo), en aquellos alumnos con valoraciones medias o altas en los autoconceptos Social y Físico, y por debajo del promedio en las categorías Académica y Emocional.

Evidentemente, en razón de los diferentes análisis y razonamientos realizados en los apartados de regresión logística (3.3) y curva ROC (3.4), es posible sostener que el test AF5 es un instrumento que posee validez explicativa, predictiva y discriminante respecto del rendimiento académico, en especial cuando está involucrado el conjunto de dimensiones que participan como covariables del modelo.

\section{4.- DISCUSIÓN Y CONCLUSIONES}

En la presente investigación nos habíamos propuesto lograr, en el marco de una comunidad de estudiantes universitarios de primer año, el desarrollo de un modelo de predicción logística que permita explicar las relaciones existentes entre distintas áreas del autoconcepto y el rendi- 
miento académico. Pues bien, a fin de alcanzar el objetivo planteado se efectuaron diversos procedimientos estadísticos los cuales han posibilitado evaluar la fiabilidad y validez del instrumento empleado, así como comprender las principales características que presentaría la asociación entre el constructo objeto de estudio y los resultados educativos de los jóvenes participantes.

En efecto, a partir de los estudios iniciales (estadísticos descriptivos, correlación dimensión-total corregida y alfa de Cronbach) realizados sobre las dimensiones del test utilizado, así como de los análisis implementados posteriormente (correlacionales y de regresión), ha sido posible comprobar: a) que la prueba aplicada constituye un instrumento confiable y válido para medir la percepción que los estudiantes tienen acerca de ellos mismos en distintos aspectos, y b) la manera en que se vinculan los tipos de autoconcepto estudiados con el rendimiento cognitivo en el contexto de la asignatura CB.

Así pues, en relación con la fiabilidad de la prueba, los resultados indican que puede considerarse un instrumento aceptable, dado que el coeficiente de consistencia interna encontrado para el conjunto de las cinco dimensiones $(\alpha=.71)$ supera el valor mínimo requerido $(\alpha=.70)$. A su vez, como complemento de la información dada, podemos decir que las correlaciones entre cada categoría y la $\mathrm{AF}_{5}$ (denominado índice de homogeneidad corregido) fueron siempre muy razonables, en todos los casos superan el valor de referencia .20 (van de $r_{d-t}=.35$ a $r_{d-t}=.59$ ). También los indicadores $\alpha$ de Cronbach obtenidos (cuando se excluye la dimensión) serían correctos para un estudio de estas características.

En razón de los resultados conseguidos en el estudio de validez predictiva, nuestra apreciación respecto de los niveles de discriminación -mediante las categorías de la prueba- de los resultados educativos es lógicamente favorable; esto es, pensamos que la AF5 es un instrumento que clasifica adecuadamente a los estudiantes con diferentes grados de logro académico. Así por ejemplo, utilizando el modelo obtenido en el apartado de regresión logística, se podría explicar y predecir que los alumnos que posean valoraciones altas en las dimensiones Académica y Emocional (sin que también lo sean en las dos dimensiones restantes, Social y Física), tendrían mejores resultados cognitivos en la asignatura objeto de interés. Por el contrario, en aquellos estudiantes con valoraciones bajas en las áreas Académica y Emocional (y quizás altas en las otras dos dimensiones), se observaría un menor rendimiento en el campo de conocimiento bajo análisis.

Aunque en su generalidad, los resultados muestran evidencia que el test aplicado presenta suficientes bondades para ser utilizado en la evaluación de las formas de autoconcepto, así como en el dominio de la inferencia estadística causal, ya que posibilitó modelizar el rendimiento académico en el ámbito de una asignatura del área Contable, creemos necesario considerar algunas limitaciones.

En efecto, en primer lugar, los participantes de la presente investigación fueron alumnos de primer año de una unidad académica específica, lo que no permitiría hacer inferencias demasiado generales sobre otros estudiantes universitarios o extender los resultados obtenidos sobre poblaciones no representadas en la muestra. 
En segundo orden, no se puso a prueba el instrumento AF5 en función de: a) variables demográficas como la edad y el género de los participantes, y b) regímenes de promoción y modalidad de cursado en la que están inscriptos los jóvenes encuestados, por lo que sería interesante en próximos estudios, analizar en el ámbito de aplicación del test cómo se manifiestan los tipos de autoconcepto al considerar algunos de estos aspectos.

Sin embargo, a pesar de las limitaciones expuestas, por lo que los resultados logrados deberían aceptarse con cierta cautela, pensamos que el trabajo realizado debe ser reconocido como un paso adelante en el abordaje del tema objeto de interés y, consecuentemente, un aporte a la comunidad académica y científica del área de conocimiento, con posibles proyecciones en política, planificación y gestión educativa, de allí que el presente estudio conlleva implícitamente verdaderas perspectivas de transferencia.

Como última reflexión se indica que el hecho de haber validado empíricamente el AF5 (a efectos de utilizarlo en la modelización de los resultados educativos) en un determinado contexto académico y sociocultural, da origen a contar con un nuevo marco de referencia, lo cual permite ampliar la aplicación de la prueba objeto de análisis; en esta oportunidad, utilizando una muestra conformada por estudiantes de Ciencias Económicas con residencia en la zona noreste de Argentina. Por lo que antecede, se considera que tanto la temática desarrollada como el tratamiento realizado constituyen un aporte científico genuino en razón de la producción de saberes que fue posible generar a partir de datos correspondientes a nuestro lugar de pertenencia, que no habían sido relevados en trabajos anteriores.

Desde nuestro punto de vista, el autoconcepto en sus distintas formas representa una cuestión relevante por su implicancia en el rendimiento académico, por lo que deberían incrementarse sus líneas de investigación a efectos de lograr un mayor desarrollo sobre su conocimiento y utilidad en nuestro ámbito de actividades. Este hecho, evidentemente, sería una importante contribución al proceso de enseñanza-aprendizaje de la asignatura bajo estudio, y de otras que se encuentran en la misma área de conocimiento, puesto que daría lugar a sugerir estrategias de mediación psicopedagógicas, tanto preventivas como correctivas, con el propósito principal de alcanzar un mejor desempeño cognitivo de los estudiantes en el espacio educativo objeto de intervención.

\section{REFERENCIAS BIBLIOGRÁFICAS}

American Psychological Association (2009). Publication Manual of the American Psychological Association (6th. ed.). Washington DC: Author.

Burns, R. (1982). Self-concept development and education. London: Holt, Rinehart \& Winston. 
Doron, R. y Parot, F. (1998). Diccionario Akal de Psicología. Madrid: Akal.

Escurra, L., Delgado, A., Guevara, G., Torres, M., Quezada, R., Morocho, J., Rivas, G. y Santos, J. (2005). Relación entre el autoconcepto de las competencias, las metas académicas y el rendimiento en alumnos universitarios de la ciudad de Lima. Revista de Investigación en Psicología, 8(1), 87-106.

García, F. y Musitu, G. (2009). AF5. Autoconcepto Forma 5 (3a. ed.). Madrid: TEA.

Harter, S. (1986). Psychological perspective on the self. Hillsdale, NJ: Erlbaum.

Huth, J., Delorme, D. y Reid, L. (2006). Perceived third-person effects and consumer attitudes on preventing and banning DTC advertising. Journal of Consumer Affairs, 4O(1), 90-116.

Jovell, A. (1995). Análisis de regresión logística. Madrid: Centro de Investigaciones Sociológicas.

Kline, P. (2000). The handbook of psychological testing (2nd. ed.). London: Routledge.

Markus, H. y Wurf, E. (1987). The dynamic self-concept: social psychological perspective. Annual review of psychology, 38, 299-337.

Marsh, H. (1993). Academic self-concept: Theory measurement and research. En J. Suls (Ed.), Psychological perspectives on the self(Vol. 4, pp. 59-98). Hillsdale, NJ: Erlbaum.

Nunnaly, J. y Bernstein, I. (1994). Psychometric theory (3rd. ed.). New York: McGraw-Hill.

Núñez, J. y González, J. (1994). Determinantes del rendimiento académico. Variables cognitivo-motivacionales, atribucionales, uso de estrategias y autoconcepto. Oviedo: Servicio de Publicaciones de la Universidad de Oviedo.

Núñez, J., González, J., García, M., González, S., Roces, C., Álvarez, L. y González, M. (1998). Estrategias de aprendizaje, autoconcepto y rendimiento académico. Psicothema, 10(1), 97-109.

Real Academia Española (2014). Diccionario de la lengua española (23a. ed.). Madrid: Espasa-Calpe.

Shavelson, R., Hubner, J. y Stanton, G. (1976). Validation of construct interpretations. Review of Educational Research, 46, 407-441. 
ANTONIO H. CLOSAS - NOELIA B. FRANCHINI - LUCIANA CYNTHIA KUC - MARÍA ALICIA DUSICKA - CARLOS M. HISGEN

$>206$ MODELO LOGÍSTICO EXPLICATIVO DE LAS RELACIONES ENTRE AUTOCONCEPTO Y RENDIMIENTO ACADÉMICO Págs. 187 - 208. DOI: http://dx.doi.org/10.30972/rfce.0203261

Suls, J. (1982). Psychological perspectives on the self(Vol. 1). Hillsdale, NJ: Erlbaum.

Suls, J. y Greenwald, A. (1983). Psychological perspectives on the self (Vol. 2). Hills-dale, NJ: Erlbaum.

Thompson, B. (1994). Guidelines for authors. Educational and Psychological Measurement, $54,837-847$.

\section{COMO CITAR ESTE ARTÍCULO:}

CLOSAS, Antonio Humberto - FRANCHINI, Noelia Beatriz - KUC, Luciana Cynthia - DUSICKA, María Alicia - HISGEN, Carlos Matías. Modelo logístico explicativo de las relaciones entre autoconcepto y rendimiento académico. Revista de la Facultad de Ciencias Económicas - UNNE. Número 20, otoño 2018, ISSN 1668 - 6365. Págs. 187 - 208. DOI: http://dx.doi. org/10.30972/rfce.0203261

\section{CURRICULUM VITAE}

\section{Antonio Humberto Closas}

Se desempeña como Profesor Titular de Estadística II y Director de Proyectos de Investigación en la Facultad de Ciencias Económicas de la Universidad Nacional del Nordeste, esta última función también la ejerce en la Facultad Regional Resistencia de la Universidad Tecnológica Nacional. Ha obtenido el grado de Doctor, mención “cum laude”, en la Universidad Pública de Navarra, España, en el área de Estadística e Investigación Operativa. Sus líneas de investigación están asociadas con métodos estadísticos multivariantes aplicados a estudios sociales y económicos. Es coautor de libros y de diversas publicaciones en revistas de impacto científico nacionales y extranjeras, algunas de las cuales se encuentran reconocidas en el Social Sciences Citation Index. Es investigador categorizado del Programa de Incentivos, de la Secretaría de Políticas Universitarias, del Ministerio de Educación de la Nación, así como de la Secretaría de Ciencia, Tecnología y Posgrado de la Universidad Tecnológica Nacional. Participa como conferencista y expositor en reuniones académicas que se realizan tanto en Argentina, como en el exterior, relacionadas con su especialidad. Asimismo, es integrante de distintos comités de arbitraje de revistas científicas, también actúa como director o miembro de comisiones de seguimiento y evaluación de tesis de maestrías y doctorados.

hclosas@hotmail.com 


\section{Noelia Beatriz Franchini}

Se desempeña como auxiliar docente de primera categoría en la materia Contabilidad Básica (con dedicación simple) y Organización Administrativa (con dedicación exclusiva), dictadas ambas en la Facultad de Ciencias Económicas de la UNNE. Ha obtenido la Especialización en Contabilidad Superior y Auditoria, en la Universidad Nacional del Nordeste. Integra un equipo de investigación sobre métodos estadísticos multivariantes aplicados a estudios sociales. Es coautora de publicaciones en revistas de impacto científico nacionales. Ha participado en actividades de extensión como codirectora de proyectos sociales y como expositora en talleres sobre educación en el marco del Programa permanente de tutorías universitarias de la UNNE. noeliabfranchini@gmail.com

\section{Luciana Cynthia Kuc}

Se desempeña como auxiliar docente de primera categoría con dedicación simple en las asignaturas Contabilidad Básica y Estados Contables de la Carrera de Contador Público, que se dicta en la Facultad de Ciencias Económicas, de la Universidad Nacional del Nordeste. Ha obtenido el título de Especialista en Contabilidad Superior y Auditoría. Forma parte de un equipo de investigación que trabaja sobre métodos estadísticos multivariantes aplicados a estudios sociales y económicos. Es coautora de diversas publicaciones. Es investigadora categorizada del Programa de Incentivos, de la Secretaría de Políticas Universitarias, del Ministerio de Educación de la Nación. lucianakuc@hotmail.com

\section{María Alicia Dusicka}

Profesional con experiencia avalada en el medio, realizando funciones de Gestión (Consultor Senior Categoría II y III seleccionada por el Banco Mundial, Secretaria Administrativa de Facultad, Secretaria de Departamento de Administración, Auditoría Externa, entre otros); de Asesoramiento; de Coordinación y de manejo de equipos unidisciplinarios como interdisciplinarios; además de gestión política en el Consejo Profesional de Ciencias Económicas del Chaco. Antigüedad con 25 años en la Docencia Universitaria, ingresando por concurso, en asignaturas del área de Administración UNNE. Integrante en jurados y comisiones evaluadoras. Docente Investigador categorizado del Programa de Incentivos de la Secretaría de Políticas Universitarias, del Ministerio de Educación de la Nación, desde el año 2013. Integrante de un equipo multidisciplinar de investigación de modelos estadísticos aplicados a estudios sociales.

mad2607@yahoo.com

\section{Carlos Matías Hisgen}

Se desempeña como Profesor Titular de Econometría, Jefe de Trabajos Prácticos de Estadística II e Investigador en la Facultad de Ciencias Económicas de la Universidad Nacional del Nordeste. Sus estudios académicos incluyen una Licenciatura en Economía, cursos de post- 
grado del Master en Economía de la Universidad de San Andrés y el grado de Magíster en Técnicas Estadísticas de la Universidad de Santiago de Compostela, España. Sus líneas de investigación relacionan la inferencia causal con modelos de regresión paramétricos y no paramétricos, dentro de las áreas de Economía de la Educación, Economía Industrial y Desarrollo Económico. Es investigador categorizado del Programa de Incentivos, de la Secretaría de Políticas Universitarias, del Ministerio de Educación de la Nación.

mhisgen@gmail.com 